\title{
Commentary - Do the negative results of the PENUT trial close the book on erythropoietin for premature infant brain?
}

\author{
Joseph J. Volpe ${ }^{\mathrm{a}, \mathrm{b}, *}$ \\ ${ }^{a}$ Department of Neurology, Harvard Medical School, Boston, MA, USA \\ ${ }^{\mathrm{b}}$ Department of Pediatric Newborn Medicine, Harvard Medical School, Boston, MA, USA
}

Received 26 March 2020

Accepted 5 April 2020

Keywords: Erythropoietin, prematurity, neurodevelopmental outcome, pre-oligodendrocytes

\section{Introduction}

Erythropoietin (EPO), long-recognized for its erythropoietic effects, has been shown in multiple animal models of hypoxic-ischemic and inflammatory brain injury to have major "neuroprotective" effects $[1,2]$. In such models of human premature brain injury a particular focus on prevention of pre-oligodendrocyte (pre-OL) death and consequent white matter injury (WMI) has been appropriate, because WMI is the central neuropathology in such infants [3]. EPO exerts its neuroprotective effects by anti-excitotoxic, antioxidant, anti-inflammatory, and anti-apoptotic effects $[1,2]$. EPO has been shown specifically to prevent or mitigate WMI in excellent animal models [4-7].

Stimulated by the apparent experimental benefit of EPO, numerous clinical studies assessing a role for EPO have been carried out [8]. A meta-analysis of

*Address for correspondence: Joseph J. Volpe, M.D., Department of Pediatric Newborn Medicine, Brigham and Women's Hospital, 221 Longwood Avenue, Room 343 C, Boston, MA 02115 USA. Tel.: +1 617525 4145; E-mail: Joseph.volpe@childrens.harvard.edu. four randomized controlled trials comprising 1133 infants indicated that prophylactic EPO administration reduced the incidence of mental developmental index scores of $<70$ (odds ratio 0.51 ( 0.31 to 0.81 ), $p<0.005$ ) at $18-24$ months [8]. Notably, in one study MRI at term equivalent age showed decreased WMI and better white matter maturation in EPO-treated infants $[9,10]$. However, study designs differed among the trials, including especially randomization, numbers of infants at early gestational ages, and durations of treatment. Because the total numbers of infants less than 28 weeks' gestational age were not large enough to assess adequately the outcome in this critical group and because these extremely preterm infants have the highest risk of unfavorable outcomes, more data were clearly needed.

\section{PENUT trial}

On this background a recent multicenter (19 sites, 30 hospitals), randomized, double-blind trial of EPO was undertaken in 741 infants ( 24 to $27^{6 / 7}$ weeks' 
gestation) [PENUT trial], i.e., preterm EPO Neuroprotection] [11]. Infants were administered EPO or placebo within 24 hours of birth and continued through 32 weeks' gestational age. Doses were chosen in part on the basis of experimental studies that indicate that "neuroprotective" effects of EPO require doses considerably higher than those needed to achieve erythropoietic effects [12]. The results of this carefully executed trial were disappointing. Thus, no differences between the EPO and placebo groups were observed at 22 to 26 months of age in the primary outcome of death or severe neurological impairment ( $26 \%$ vs. $26 \%$ ), or in the key secondary outcome of death or moderate to severe neurodevelopmental impairment (48\% vs. 47\%). A limitation of the study, explicitly acknowledged by the investigators [11], is that cognitive testing at two years of age is not so reliable as testing at later ages [13]. Thus, a metaanalysis of 24 studies in which early assessments (by Bayley Scales of Infant Development-III or Griffiths Scales of Mental Development) performed at $1^{1 / 2}$ to 3 years of age were compared with later cognitive tests carried out at school age showed that the pooled sensitivity of early assessments for identifying school-age cognitive deficits was only $55 \%$. Thus, later assessment of the infants in the PENUT trial will be important. Additionally, because EPO has particularly beneficial effects on pre-OLs and, thereby, potentially white matter development and because the latter is an active process well beyond infancy and thereby crucial for cognitive development, the appearance of deficits in specific cognitive functions, e.g., executive function, at later ages is a critical possibility to be tested. Notably, the EPO regimen in the PENUT trial did show stimulation of erythropoiesis, and no serious adverse effects.

Do the results of the large PENUT trial close the book on the question of any value for EPO in improving neurological outcomes in extremely preterm infants? The purpose of this Commentary is to suggest that this study should not close the book on EPO but, rather, should raise the possibility that the duration of EPO treatment was too brief.

\section{Neuroprotection vs neurorestoration}

"Neuroprotection" is a term often used to refer to protection from cell death in the central nervous system. In models of hypoxic-ischemic disease or stroke, the term refers primarily to protection from neuronal death. In the case of the premature infant the term is most often used to refer primarily to death of pre-OLs, the principal cellular target in cerebral WMI of prematurity [14]. In experimental models of WMI, EPO has been shown to prevent pre-OL death [4-7]. However, it has been well-established in studies of WMI in experimental models and in human premature brain that after the initial pre-OL death, proliferation of oligodendroglial progenitors and replenishment of the pre-OL pool occur [3, 15, 16]. A subsequent failure of maturation of these pre-OLs to mature OLs that ensheath axons and form myelin is the central disturbance in WMI of prematurity. This failure leads to the subsequent dysmaturational events involving both white and gray matter structures that underlie the subsequent neurological deficits in these infants [17]. The failure of pre-OL maturation and subsequent dysmaturational events occur over many weeks to months after the initial injury [18]. These dysmaturational events are mediated to a major degree by the prominent microgliosis and astrogliosis that are the hallmarks of subacute to chronic WMI. As reviewed elsewhere [19], in WMI microglia and astrocytes, normally crucial for a variety of aspects of normal brain development, become "activated" (microglia) and "reactive" (astrocytes). This change from a normal to an inflammatory phenotype results in molecular events that impair pre-OL maturation [19]. This diffuse white matter gliosis is present in the brain of premature infants with WMI for many weeks and likely, months after the neonatal period [15, 20-22].

Neurorestorative interventions are designed to counteract these critical dysmaturational events involving the pre-OL and, likely, secondarily, such neuronal-axonal structures as cerebral cortex and thalamus $[18,23]$. Indeed, EPO has been shown to promote pre-OL development in multiple models of WMI [1, 5, 7, 24]. Promotion of angiogenesis and neurogenesis also may occur $[1,25]$. Because the failure of pre-OL differentiation is important in the genesis of axonal and neuronal dysmaturation, promotion of pre-OL differentiation by EPO would be expected to lead to widespread beneficial effects on brain maturation, as shown in experimental models [25]. The mechanisms of the beneficial effects of EPO on pre-OL maturation have been reviewed in detail elsewhere [1, 3, 24-27] but involve direct effects on pre-OLs as well as indirect effects mediated by EPO's impact on microglia and astrocytes which underlie much of the pre-OL maturational failure.

Consistent with EPO's proposed neurorestorative effects on pre-OL maturation are experimental 
studies of WMI in which EPO was administered after the period of acute cell injury. Thus, in a developing rat model of WMI induced by hypoxia-ischemia, Iwai and coworkers showed that administration of EPO at multiple time points, beginning 48 hours after the insult, led to significantly increased oligodendrogenesis and maturation of oligodendrocytes as well as attenuation of WMI [5]. In this neonatal rat model, the 48-hour delay is equivalent to many weeks to months in developing human brain. Similarly, in a combined in vivo and in vitro study, Jantzie and coworkers showed in a rat model of WMI produced by transient prenatal hypoxia-ischemia that exposure to EPO several days later leads to enhanced oligodendroglial development [24]. The findings are consistent with previous observations that following hypoxia the EPO receptor is upregulated on pre-OLs, that signaling from the vacant EPO receptor leads to pre-OL apoptosis, but that this deleterious effect can be prevented and pre-OL differentiation promoted by provision of adequate EPO ligand to occupy and activate the EPO receptor [24, 26, 27]. Taken together, the experimental data indicate value of EPO as a neurorestorative agent vis à vis the pre-OL, after the period of acute cell injury.

\section{Critical importance of duration of EPO treatment}

The duration of treatment in the PENUT trial (onset 24-28 weeks' gestational age and continued to 32 weeks' postconceptional age) "was determined on the basis of the period of oligodendrocyte vulnerability" [11]. Indeed, this period is the peak time for the occurrence of overt periventricular leukomalacia [3]. However, it now appears that modern-day WMI evolves over a much longer period and is related to the diffuse gliosis in cerebral white matter, which is present far beyond the premature period (see earlier). Moreover, although the pre-OL accounts for $90 \%$ of the oligodendroglial lineage in the extremely preterm infant, this vulnerable developing cell, even at term, still accounts for $50 \%$ of the entire oligodendroglial lineage in cerebral white matter. Thus, the vulnerable pre-OLs are still abundant at 32 weeks' postconceptional age when EPO treatment in the PENUT trial ceased. More importantly, the dysmaturational events that follow pre-OL injury, i.e. failure of pre-OL maturation, secondary dysmaturation of neuronalaxonal structures, occur over many weeks to months.
The potential importance of a relatively prolonged period of EPO treatment is suggested by a study of very preterm infants (median gestational age, 28 wks) [28]. This trial, albeit smaller $(n=99)$ than the PENUT trial, utilized EPO administration (as EPO or its higher glycosylated derivative, darbepoietin) thrice weekly through 35 weeks' postconceptual age. The treated infants had better cognitive outcomes and less neurodevelopmental impairment at age 3.5 to 4 years, when compared to placebo-treated infants (FSIQ: $91.1 \pm 17.5$ vs. $79.2 \pm 18.5, p=0.036$; performance IQ: $93.0 \pm 17.0 \mathrm{vs.} 79.5 \pm 19.5, p=0.018$ ). Thus, in this study EPO may have functioned as both a neuroprotective and neurorestorative agent.

\section{Conclusion}

The PENUT trial, in many ways, was a superb, carefully designed and intensive trial of nearly three years' duration. Repeating this trial with a longer duration of treatment, e.g., to term equivalent age or even longer, would be a major undertaking. Issues related to safety (e.g., enhanced erythropoiesis, altered iron homeostasis, etc.) would require careful consideration. However, if, as experimental data suggest, EPO is considered a potential major neurorestorative therapy, it will be difficult to conclude from the PENUT trial that this agent is not beneficial for long-term outcome in extremely preterm infants.

\section{References}

[1] Xiong T, Qu Y, Mu D, Ferriero D. Erythropoietin for neonatal brain injury: Opportunity and challenge. Int J Dev Neurosci. 2011;29:583-91.

[2] Rangarajan V, Juul SE. Erythropoietin: Emerging role of erythropoietin in neonatal neuroprotection. Pediatr Neurol. 2014;51:481-8.

[3] Kinney HC, Volpe JJ. Encephalopathy of prematurity: Neuropathology. In: Volpe JJ, Inder TE, Darras BT, deVries LS, duPlessis AJ, Neil JJ, et al., editors. Volpe's Neurology of the Newborn. Chapter 14, 6th ed. Philadelphia, PA: Elsevier; 2018. pp. 389-404.

[4] Rees S, Hale N, De Matteo R, Cardamone L, Tolcos M, Loeliger M, et al. Erythropoietin is neuroprotective in a preterm ovine model of endotoxin-induced brain injury. J Neuropathol Exp Neurol. 2010;69:306-19.

[5] Iwai M, Stetler RA, Xing J, Hu X, Gao Y, Zhang W, et al. Enhanced oligodendrogenesis and recovery of neurological function by erythropoietin after neonatal hypoxic/ischemic brain injury. Stroke. 2010;41:1032-7.

[6] Mazur M, Miller RH, Robinson S. Postnatal erythropoietin treatment mitigates neural cell loss after systemic prena- 
tal hypoxic-ischemic injury. J Neurosurg Pediatr. 2010;6: 206-21.

[7] Zhu L, Huang L, Wen Q, Wang T, Qiao L, Jiang L. Recombinant human erythropoietin offers neuroprotection through inducing endogenous erythropoietin receptor and neuroglobin in a neonatal rat model of periventricular white matter damage. Neurosci Lett. 2017;650:12-7.

[8] Fischer HS, Reibel NJ, Buhrer C, Dame C. Prophylactic early erythropoietin for neuroprotection in preterm infants: A meta-analysis. Pediatrics. 2017;139:e20164317.

[9] Leuchter RH, Gui L, Poncet A, Hagmann C, Lodygensky GA, Martin E, et al. Association between early administration of high-dose erythropoietin in preterm infants and brain MRI abnormality at term-equivalent age. JAMA. 2014;312:817-24

[10] O'Gorman RL, Bucher HU, Held U, Koller BM, Huppi PS, Hagmann CF. Tract-based spatial statistics to assess the neuroprotective effect of early erythropoietin on white matter development in preterm infants. Brain. 2015;138:388-97.

[11] Juul SE, Comstock BA, Wadhawan R, Mayock DE, Courtney SE, Robinson T, et al. A randomized trial of erythropoietin for neuroprotection in preterm infants. N Engl J Med. 2020;382:233-43.

[12] McPherson RJ, Juul SE. Recent trends in erythropoietinmediated neuroprotection. Int J Dev Neurosci. 2008;26: 103-11.

[13] Wong HS, Santhakumaran S, Cowan FM, Modi N. Developmental assessments in preterm children: A meta-analysis. Pediatrics. 2016;138:e20160251.

[14] Volpe JJ, Kinney HC, Jensen FE, Rosenberg PA. The developing oligodendrocyte: Key cellular target in brain injury in the premature infant. Int J Dev Neurosci. 2011;29:423-40.

[15] Buser JR, Maire J, Riddle A, Gong X, Nguyen T, Nelson K, et al. Arrested preoligodendrocyte maturation contributes to myelination failure in premature infants. Ann Neurol. 2012;71:93-109.

[16] Back SA. White matter injury in the preterm infant: Pathology and mechanisms. Acta Neuropathol. 2017;134:331-49.

[17] Volpe JJ. Brain injury in premature infants: A complex amalgam of destructive and developmental disturbances. Lancet Neurol. 2009;8:110-24.
[18] Volpe JJ. Dysmaturation of premature brain: Importance, cellular mechanisms and potential interventions. Pediatr Neurol. 2019;95:42-66.

[19] Volpe JJ. Microglia: Newly discovererd complexity could lead to targeted therapy for neonatal white matter injury and dysmaturation. J Neonat-Perinat Med. 2019;12:239-42.

[20] Deguchi K, Oguchi K, Takashima S. Characteristic neuropathology of leukomalacia in extremely low birth weight infants. Pediatr Neurol. 1997;16:296-300.

[21] Okoshi Y, Itoh M, Takashima S. Characteristic neuropathology and plasticity in periventricular leukomalacia. Pediatr Neurol. 2001;25:221-6.

[22] Pierson CR, Folkerth RD, Billards SS, Trachtenberg FL, Drinkwater ME, Volpe JJ, et al. Gray matter injury associated with periventricular leukomalacia in the premature infant. Acta Neuropathol. 2007;114:619-31.

[23] Volpe JJ. Brain injury in premature infants: a complex amalgam of destructive and developmental disturbances. Lancet Neurol. 2009;8:110-24.

[24] Jantzie LL, Miller RH, Robinson S. Erythropoietin signaling promotes oligodendrocyte development following prenatal systemic hypoxic-ischemic brain injury. Pediatr Res. 2013;74:658-67.

[25] Jantzie LL, Corbett CJ, Firl DJ, Robinson S. Postnatal erythropoietin mitigates impaired cerebral cortical development following subplate loss from prenatal hypoxiaischemia. Cereb Cortex. 2014.

[26] Genc K, Genc S, Baskin H, Semin I. Erythropoietin decreases cytotoxicity and nitric oxide formation induced by inflammatory stimuli in rat oligodendrocytes. Physiol Res. 2006;55:33-8.

[27] Kato S, Aoyama M, Kakita H, Hida H, Kato I, Ito T, et al. Endogenous erythropoietin from astrocyte protects the oligodendrocyte precursor cell against hypoxic and reoxygenation injury. J Neurosci Res. 2011;89:1566-74.

[28] Ohls RK, Cannon DC, Phillips J, Caprihan A, Patel S, Winter S, et al. Preschool assessment of preterm infants treated with darbepoetin and erythropoietin. Pediatrics. 2016;137:1-9. 